\title{
Se hace camino al andar: Educación médica de pregrado en el Departamento de Cirugía, Universidad de La Sabana
}

\author{
The path is made by walking: Undergraduate medical education in \\ the Department of Surgery, Universidad de La Sabana
}

\author{
Luis Carlos Domínguez-Torres ${ }^{1} \mathbb{D}$, Neil Valentín Vega-Peña ${ }^{2} \mathbb{D}$, Juan José Pepín-Rubio ${ }^{3} \mathbb{D}$, \\ Diego Orlando Sierra-Barbosa ${ }^{4} \mathbb{D}$, Juan David Lotero ${ }^{5} \mathbb{D}$
}

1 MD., MSc., MPHE., especialista en Cirugía general, Clínica Universitaria de La Sabana; profesor, Departamento de Cirugía, Universidad de La Sabana, Chía, Colombia.

2 MD., MSc., especialista en Cirugía general; coordinador, Departamento de Cirugía, Universidad de La Sabana, Chía, Colombia.

3 MD., especialista en Cirugía general, Clínica Universitaria de La Sabana; profesor, Departamento de Cirugía, Universidad de La Sabana, Chía, Colombia.

4 MD., MSc., especialista en Cirugía general, Clínica Universitaria de La Sabana; profesor, Departamento de Cirugía, Universidad de La Sabana, Chía, Colombia.

5 MD, especialista en Cirugía general, Clínica Universitaria de La Sabana, Chía, Colombia.

"Si quieres llegar rápido, ve solo. Si quieres llegar lejos, ve acompañado".

Proverbio Africano

\begin{abstract}
Resumen
La educación médica en cirugía en Colombia esta experimentando una acelerada transformación, no obstante, la documentación de esta transformación educativa en el país es limitada. En este estudio se presenta una revisión cronológica de la enseñanza en el Departamento de Cirugía de la institución. La revisión se focaliza en las estrategias e hitos alcanzados en el pregrado, luego de transformar el modelo de enseñanza tradicional de principios de siglo XXI. La revisión está basada en la síntesis de una serie de estudios originales, los cuales ofrecen evidencia sobre las intervenciones realizadas en el departamento, y sobre sus resultados. Finalmente, se presenta una reflexión en torno a los retos futuros.
\end{abstract}

Palabras clave: educación; cirugía; estudiantes de medicina; aula invertida; Colombia

Fecha de recibido: 05/05/2020 - Fecha de aceptación: 10/07/2020

Correspondencia: Luis Carlos Domínguez. Campus del Puente del Común, Km. 7, Autopista Norte de Bogotá, Chía, Colombia. Teléfono: 8615555 / 861 6666. Correo electrónico: carlosdot@unisabana.edu.co

Citar como: Domínguez LC, Valentín-Vega NV, Pepín JJ, Sierra DO, Lotero JD. Se hace camino al andar: Educación médica de pregrado en el Departamento de Cirugía, Universidad de La Sabana. Rev Colomb Cir. 2021;36:25-34. https://doi.org/10.30944/20117582.648 Este es un artículo de acceso abierto bajo una Licencia Creative Commons - BY-NC-ND https://creativecommons.org/licenses/by-ncnd/4.0/deed.es 


\begin{abstract}
Surgical education in Colombia is undergoing an accelerated transformation, however, the documentation of this educational transformation in the country is limited. This study presents a chronological review of teaching in the Department of Surgery of the institution. The review focuses on the strategies and milestones achieved in undergraduate level, after transforming the traditional teaching model of the early 21st century. The review is based on the synthesis of a series of original studies, which offer evidence on the interventions conducted in the department, and on their results. Finally, a reflection on future challenges is presented.
\end{abstract}

Keywords: education; surgery; medical students; flipped classroom; Colombia

\section{Introducción}

Durante la última década ha sido creciente el interés de la comunidad quirúrgica por la enseñanza interactiva en el pregrado ${ }^{1-3}$. Este fenómeno obedece a las limitaciones evidenciadas con la educación medica tradicional, centrada en la transmisión de información (usualmente a través de clases magistrales), para afrontar los desafíos del cuidado y seguridad del paciente, que exigen capacidad analítica y pensamiento critico de los profesionales ${ }^{4,5}$. La educación quirúrgica colombiana no es ajena a esa realidad. En el presente artículo revisamos la experiencia educativa del Departamento de Cirugía de la Universidad de la Sabana, Chía, Colombia, durante el presente siglo. En este recorrido cronológico, describimos las características de la enseñanza durante la primera década y, posteriormente discutimos cómo ha madurado el modelo de enseñanza interactiva entre los años 2012 y 2020. Por ultimo, presentamos los retos que afrontaremos en el futuro.

\section{Enseñanza quirúrgica en la primera década del siglo XXI}

Durante este periodo, la enseñanza de la cirugía en el pregrado se sustentó en estrategias tradicionales de enseñanza en el aula, específicamente clases magistrales, a través de las cuales se entregaba el contenido curricular por sistemas orgánicos a los estudiantes. Adicionalmente, los estudiantes rotaban por diversas instituciones afiliadas a la facultad de medicina para realizar su práctica clínica. La evaluación de desempeño estudiantil se centraba en el "saber", por lo general mediante exámenes de selección múltiple y orales, de acuerdo con el contenido programático.
Este modelo de enseñanza de la alta transmisión de información y la baja interactividad en el aula, frente a los crecientes retos del aprendizaje en el sitio de trabajo, fue revisado en el año 2012. Se identificaron oportunidades de mejoramiento que indicaron la necesidad de cambios orientados a:

1) actualizar el currículo al cambio demográfico, epidemiológico y tecnológico en cirugía a nivel global y local;

2) transformar el modelo de enseñanza, altamente memorístico y "centrado en el profesor", hacia uno analítico y crítico, "centrado en el estudiante";

3) incluir tecnologías de la información en la enseñanza;

4) optimizar el tiempo asignado a las clases magistrales para dinamizar el aprendizaje interactivo y participación estudiantil,

5) promover el desarrollo profesoral en educación médica (faculty development) y la investigación educativa; $y$

6) conducir evaluación estudiantil sumativa en niveles superiores de la pirámide de Miller, para informar sobre el desempeño integral del estudiante en dimensiones del "saber como" y el "ser" ${ }^{6}$.

\section{Enseñanza quirúrgica actual (2012 - 2020)}

A partir de las necesidades identificadas, realizamos cambios profundos en nuestro modelo de enseñanza, congruentes con el proyecto educativo 
institucional. Los principales desarrollos se encuentran en la enseñanza interactiva adaptada al contexto de práctica. Nuestro objetivo principal privilegia la capacidad analítica del estudiante para la resolución de problemas cotidianos.

Una de las principales estrategias que hemos utilizado es la de aula invertida. Esta estrategia constructivista invierte los componentes habituales de una clase, y los distribuye en actividades "fuera del aula" en forma de videos y otros recursos (por ejemplo, documentos, podcast) enfocados en el aprendizaje independiente y, posteriormente emplea el tiempo "en el aula física" para ejercicios de aprendizaje interactivo (por ejemplo, discusiones de caso y ejercicios para la resolución de problemas) ${ }^{4,5}$. Con estas actividades pretendemos que el estudiante desarrolle habilidades cognitivas superiores para el análisis, aplicación y evaluación de información, en vez de memorizar información. Por lo tanto, buscamos trasladar eficientemente la teoría quirúrgica a la práctica para promover la comprensión, y aprovechar el recurso humano y tecnológico disponible.

Estos cambios han requerido esfuerzos en materia de investigación educativa para documentar nuestra experiencia, así como desarrollo profesoral permanente en el campo de la educación para profesiones de la salud. Igualmente, han requerido la participación de profesores clínicos (internistas, neurólogos, patólogos y profesores de simulación clínica).

A continuación, presentamos los desarrollos y los resultados más importantes en este periodo.

\section{a. Principales desarrollos}

\section{Aula invertida convencional}

En 2012 actualizamos el listado de competencias (conocimientos, habilidades y actitudes) que deben demostrar los estudiantes de medicina en cirugía. Con esta información, desarrollamos a partir de 2013 un curso de 18 semanas, en formato de aula invertida convencional, en el que las actividades se dividen en dos fases: virtual y presencial ${ }^{6,7}$. En la primera, los estudiantes revisan de manera independiente la información referente a cada tema (por ejemplo, patología quirúrgica de la vesícula y vía biliar), a través de videos (en formato de clases magistrales virtuales), artículos, información complementaria y foros de discusión. Posteriormente, los estudiantes resuelven individualmente 4-6 casos clínicos por semana.

La segunda fase, o presencial, tiene lugar en el aula "física" de clase. En sesiones presenciales de tres horas a la semana se utiliza la metodología de discusión basada en casos, con base en aquellos casos revisados previamente por los estudiantes. Primero, durante 30 minutos, se realizan discusiones interactivas en grupos pequeños de trabajo (en promedio de 10 estudiantes), y después se realiza una discusión plenaria. En cada sesión presencial, las discusiones son facilitadas por cuatro profesores de cirugía.

También hemos utilizado este formato convencional de aula invertida para la enseñanza de habilidades y actitudes orientadas al trabajo en equipo entre estudiantes y profesores ${ }^{8,9}$, así como para la enseñanza articulada de humanidades médicas y cirugía ${ }^{10}$. El componente práctico del curso de cirugía se realiza en servicios de cirugía de instituciones públicas y privadas.

\section{Extensión del aula invertida a simulación clínica}

En el año 2016 desarrollamos una estrategia educativa para el manejo del paciente traumatizado bajo el modelo 4C/ID (cuatro componentes de diseño instruccional), con base en tareas de aprendizaje, información de soporte, información procedimental y tareas prácticas ${ }^{11}$.

Identificamos la necesidad de incluir una fase práctica en el aula invertida para el desarrollo de habilidades específicas, en congruencia con los principios del Curso ATLS ${ }^{\circledR}$ (Advanced Trauma Life Support). Por esta razón, realizamos una variación al modelo convencional de aula invertida, el cual denominamos aula invertida extendida (AIE), incorporando una tercera fase en la que las actividades presenciales se extienden al laboratorio de simulación ${ }^{9,12}$. Con esta fase pretendemos que actividades de simulación de mediana fidelidad complementen los componentes virtual y presencial, orientados a la adquisición de información y resolución de casos clínicos. 
Las tareas prácticas en el ambiente simulado se desarrollan en estaciones de trabajo supervisadas por profesores de cirugía y anestesiólogos, que están enfocadas en: 1) revisión primaria y resucitación; 2) manejo de vía aérea e inmovilización cervical; 3) manejo de las lesiones torácicas; 4) manejo del shock y acceso vascular; 5) revisión de estudios radiológicos (radiografías básicas y tomografía), y 6) avance de sondas ${ }^{12}$.

Igualmente, hemos utilizado el modelo de AIE para la enseñanza de principios de cirugía mínimamente invasiva (CMI) a estudiantes de medicina. Con este propósito desarrollamos otro curso en 2017, en cuya fase virtual se expone la fundamentación teórica, y en la presencial convencional se discuten casos clínicos relacionados. La tercera fase se desarrolla en el laboratorio de simulación a través de cuatro estaciones de trabajo: 1) liderazgo, comunicación, proactividad y colaboración enfocadas a la seguridad del paciente; 2 ) funcionamiento y posición de la torre de laparoscopia / funcionamiento de la cámara; 3) instrumental y ejercicios básicos de transferencia, profundidad, corte y precisión en pelvi-trainer y, 4) principios de anudado y sutura básica en especímenes ${ }^{9}$.

El propósito de la evaluación estudiantil en estas dos estrategias (AIE para el manejo del paciente traumatizado y AIE para la enseñanza de CMI) se centra en la demostración de «saber cómo» (know how) y «demostrar cómo» (show how), de acuerdo con la Pirámide de Miller. Por esta razón, las evaluaciones incorporan no solo pruebas escritas, sino también evaluaciones practicas mediante examen clínico objetivo estructurado (ECOE). Ambos cursos continúan activos en el currículo.

\section{Recursos adicionales para el aprendizaje}

Hemos incorporado estrategias orientadas a promover el aprendizaje en el entorno virtual y presencial del aula. En el primero, nos hemos focalizado en desarrollar videos concretos que cuenten con información suficiente, y cuya extensión no sea superior a 10 minutos. Estos recursos son alojados en una plataforma institucional. El curso básico de cirugía cuenta con cerca de 60 videos y cada curso extendido a simulación clínica con 6 videos. Adicionalmente, se desarrollaron 65 casos clínicos originales, cubriendo las patologías mas frecuentes de la cirugía, los cuales tienen en cuenta el contexto de práctica colombiano, la epidemiologia local y los recursos disponibles, y cerca de 40 casos originales para la integración de humanidades y cirugía. Finalmente, se incluyeron listas de chequeo para el autoestudio, con base en las competencias esperadas.

También se han desarrollado nuevas herramientas en la fase presencial. A nuestro juicio, esta es la fase más importante del modelo pues brinda la oportunidad de interactuar y desarrollar habilidades cognitivas superiores (analizar, crear, evaluar). Uno de los recursos que se ha implementado es el mapa conceptual para la síntesis de información, resultante del componente virtual y la discusión presencial. Somos conscientes que la información del aula invertida es mayor que la de estrategias tradicionales (clases magistrales). Este fenómeno es esperable porque los estudiantes no solo tienen que revisar contenidos fundamentales, sino también aplicarlos para la resolución de problemas en casos clínicos. La cantidad de información, por lo tanto, puede crear confusión y dificultad para trasladar la teoría a la práctica. Los mapas conceptuales, tras la discusión de cada caso clínico, nos han permitido superar estos desafíos, sintetizar la información y puntualizar los conceptos mas importantes ${ }^{13}$.

Se diseñaron diversas formas de división de cada cohorte (en promedio de 60 estudiantes) en grupos pequeños, con el fin de promover el aprendizaje durante las discusiones de casos y de mejorar el clima de aprendizaje ${ }^{14}$. Varios de estos aspectos prácticos, resultantes de nuestra experiencia, han sido recopilados en recomendaciones para educadores y cirujanos ${ }^{15}$.

\section{Psicometría para la investigación educativa}

El desarrollo de estas intervenciones educativas ha requerido instrumentos con adecuadas propiedades psicométricas para la medición de la calidad programática y desenlaces educativos. Por lo tanto, se hizo la traducción y la validación de contenido y constructo de instrumentos como el Flipped 
Classroom Perception Instrument (FCPI), el cual esta enfocado en la evaluación de los componentes virtual y presencial del aula ${ }^{16}$. Los resultados del análisis factorial exploratorio (AFE), indican una estructura factorial del instrumento en español similar a la del original. También se determinó la consistencia interna de instrumentos como Dundy Ready Educational Environment Measure (DREEM), Concept Mapping Questionnaire (CMQ), Maastricht Clinical Questionnaire (MCQ), Learning Impact Questionnaire (LIQ), Self-directed Learning Readiness Scale (SDLRS), Revised Two Factor Study Process Questionnaire (R-SPQ- 2F), Teamwork Perceptions and Attitudes Questionnaires (TeamSTEPPS) y Metacognitive Awareness Inventory (MAI) ${ }^{17-24}$.

Por último, se construyeron y adaptaron instrumentos para la evaluación de la calidad del trabajo en equipo en cirugía (TECUS) y para la evaluación de la calidad académica de las rotaciones clínicas (ROTA-Q) ${ }^{25,26}$. En nuestras mediciones, la confiabilidad de estos instrumentos ha sido adecuada ${ }^{6,8-10,12-15,27}$.

\section{b. Resultados principales \\ 1. Ambiente de aprendizaje}

La evaluación general del ambiente de aprendizaje en 14 cohortes consecutivas (2013-2019), que incluyen un total de 907 estudiantes, demuestra que el aula invertida favorece un ambiente de aprendizaje positivo, según las percepciones estudiantiles y valores normativos de referencia del DREEM. El puntaje promedio del DREEM durante siete años de evaluación es $142 \pm 12,99$ (rango 120-157). Durante este periodo los puntajes de los dominios del DREEM que evalúan la enseñanza, habilidades académicas, profesores, atmosfera y ambiente social han permanecido en niveles superiores ${ }^{15}$.

De la misma forma, entre 2013 y 2014 comparamos el ambiente de aprendizaje del aula invertida con la estrategia tradicional de clases magistrales. Analizamos las respuestas de 207 estudiantes con respecto a sus propias experiencias con las dos estrategias. Identificamos que el puntaje global del DREEM, así como el de todos sus dominios, fue superior en el aula invertida (152 versus 112) $(\mathrm{p}<0,01)^{6}$. Estos hallazgos nos estimularon a realizar nuevas comparaciones con otros ambientes de aprendizaje en instituciones diferentes. Para tal fin, en 2018 comparamos el ambiente de aprendizaje de nuestra aula invertida con el de otras facultades de medicina que utilizan estrategias de enseñanza tradicional en cirugía (clases magistrales). Diseñamos un estudio pre-experimental y encontramos que el puntaje global del DREEM fue estadísticamente superior en el aula invertida $145,01 \pm 21,75(85-186)$ versus $126,64 \pm 20,89(73-187)(\mathrm{p}<0,05)^{28}$.

Por último, nuestra experiencia indica que la forma de división de grupos grandes de trabajo en grupos pequeños para el aprendizaje interactivo tiene efecto sobre el ambiente de aprendizaje. Estos resultados los observamos en un estudio en el que medimos la calidad del ambiente entre grupos pequeños de trabajo interactivo distribuidos en una misma aula física, bajo la supervisión simultánea de diferentes profesores, y la comparamos con la de grupos de trabajo distribuidos en aulas físicas independientes bajo la supervisión de un solo profesor. Nuestros resultados demostraron puntajes superiores en el DREEM cuando los estudiantes están una misma aula física y tienen varios profesores a su disposición 145,01 $\pm 21,75$ versus $131,46 \pm 18,48(\mathrm{p}<0,05)$.

\section{Aprendizaje (conceptos y habilidades) y éxito académico}

En un análisis de las percepciones de 480 estudiantes (2013-2016), identificamos una relación positiva entre el ambiente de aprendizaje del aula invertida y sus habilidades académicas $(\beta=0,43$; $\mathrm{p}<0,001)^{27}$. Igualmente, hemos encontrado efectos positivos en la percepción global de aprendizaje tras varias semanas de inmersión en el aula extendida a simulación clínica para la resucitación del paciente traumatizado ${ }^{12}$. Mediante el estudio de las percepciones de 75 estudiantes en el cuestionario FCPI, identificamos puntajes que aumentaron de $3,98 \pm 0,58(1,5-5,0)$ a $4,24 \pm 0,64(1,38-5,0)$ $(\mathrm{p}<0,05)$, demostrando un efecto moderado del AIE sobre el aprendizaje $(\mathrm{d}$ Cohen $=0,42)$. 
En tercer lugar, hemos identificado cambios significativos en los resultados de pruebas académicas de conocimiento teórico y habilidades practicas, mediante Evaluación Clínica Observacional Estructurada (ECOE), en ambientes simulados ${ }^{9,12}$. Por ejemplo, encontramos un efecto grande del AIE para incrementar el conocimiento de los estudiantes sobre los principios de CMI (d Cohen $=1,52$ ). El efecto de la intervención sobre habilidades practicas de transferencia, corte y precisión en pelvi-trainer, fue igualmente significativo ( $d$ Cohen $=2,0$ ) ${ }^{9}$.

El uso de herramientas, como los mapas conceptuales al interior del aula invertida, han contribuido significativamente $(\mathrm{p}<0.001)$ a mejorar la participación de los estudiantes en las sesiones presenciales, a comunicar su aprendizaje, a pensar independientemente mientras se aprende cooperativamente, y a realizar conexiones entre conceptos complejos. Estos resultados han sido documentados cuantitativamente mediante el cuestionario CMQ, al principio de dos cursos y tras dieciocho semanas de intervención ${ }^{13}$. Los hallazgos son similares con el uso de listas de chequeo para el aprendizaje. La tasa de éxito académico (aprobación del curso) ha sido cercana al $97 \%{ }^{15}$.

\section{Aprendizaje autodirigido}

Nuestros resultados indican adecuados niveles de auto-direccionamiento en el aprendizaje, entre diversos grupos de estudiantes, de acuerdo con el cuestionario SDRLS ${ }^{28}$. En 2018, el puntaje global de SDRLS de 75 participantes fue 3,89 $\pm 0,38^{2,8-4,7}$. No obstante, estos resultados fueron similares y no demostraron diferencias significativas, frente a los de un grupo control de estudiantes asignados a clases magistrales (ambiente tradicional) $(p=0,5)$. Pese a estos hallazgos descriptivos, encontramos que la correlación entre el ambiente de aprendizaje (DREEM) y el nivel de aprendizaje autodirigido fue superior en el aula invertida $(r=0,48)$ en comparación con la correlación observada en clases magistrales $(r=0,29)(p<0,001){ }^{28}$.

\section{Metacognición y enfoques de aprendizaje}

En dos cohortes consecutivas de estudiantes en 2017, se midieron los niveles de habilidades de los estu- diantes para aprender a aprender (metacognición), antes y después de participar en el aula invertida. De acuerdo con los dominios del cuestionario MAI, encontramos efectos positivos sobre el conocimiento declarativo (propio aprendizaje), procedimental (uso de estrategias de aprendizaje) y condicional (cuando y porque utilizar estrategias de aprendizaje). Además, identificamos efectos sobre las capacidades de los estudiantes para planificar (d Cohen $=0,31$ ) y evaluar su aprendizaje ( $\mathrm{d}$ Cohen $=0,31$ ).

Por otra parte, hemos encontrado bajos niveles de aprendizaje superficial (orientado al aprendizaje memorístico) y niveles altos de aprendizaje profundo (orientado a la comprensión), de acuerdo con el cuestionario R-SPQ- 2F, antes y después de la intervención. Aunque estos resultados son interesantes deben interpretarse cuidadosamente pues carecen de grupo control. Hasta el momento, puede inferirse que el aula invertida no ha ejercido un efecto significativo sobre el enfoque de aprendizaje individual.

\section{Trabajo en equipo}

Otro de los objetivos de nuestra estrategia ha sido promover el desarrollo de habilidades no-técnicas. Una de estas se relaciona con las actitudes positivas de los estudiantes orientadas al trabajo en equipo. Tras diversos módulos de inmersión en ambientes virtuales y en actividades extendidas al aula convencional y al laboratorio de simulación, hemos identificado cambios significativos en las actitudes estudiantiles, de acuerdo con mediciones realizadas en el cuestionario Team-STEPPS. Por ejemplo, han mejorado las actitudes positivas para el reconocimiento de situaciones críticas, monitoreo, liderazgo y comunicación en entornos simulados de CMI. En estos estudios, los puntajes del Team-STEPPS aumentaron de 3,73 $\pm 0,25$ (rango 2,82-4,0) previo a la intervención a 4,23 \pm 0,24 (rango 3,52-4,86) posterior a la intervención $(\mathrm{p}<0,05)$, y el tamaño del efecto fue grande (d Cohen =1,97). También hemos evidenciado hallazgos similares en cursos generales de trabajo en equipo, orientados a crear transformaciones positivas y concientización de este componente del cuidado clínico entre estudiantes y profesores ${ }^{8}$. 


\section{Satisfacción estudiantil}

Por ultimo, y no menos importante, el aula invertida en cirugía ha sido una estrategia de enseñanza que ha promovido alta satisfacción estudiantil al final de los cursos 9,12. Estos hallazgos han sido documentados a partir de ítems individuales del DREEM, pero también a partir de cuestionarios institucionales, que involucran calificaciones de escalas y comentarios individuales. Resaltamos, en particular, los resultados de diversos ítems que evalúan la coherencia entre los contenidos y la evaluación sumativa (blueprint), los cuales en más del $75 \%$ de las evaluaciones estudiantiles han sido superiores a 4.0 (rango 0-5) ${ }^{9,12}$. Estos hallazgos, en otras palabras, indican que lo que se evalúa es coherente con lo que se enseña.

\section{El futuro del modelo de enseñanza}

Los desarrollos y resultados expuestos contribuyen a la extensa experiencia educativa en el pregrado, desarrollada en múltiples departamentos quirúrgicos en Colombia, y a sus resultados positivos en simulación clínica, enseñanza clínica, diseño curricular, profesionalismo y persistencia académica de los estudiantes, entre otros ${ }^{29-36}$. Igualmente, contribuye al notable cuerpo de evidencia en educación de postgrado en cirugía desarrollado en el país ${ }^{37-52}$.

No obstante, nuestros desarrollos educativos en el pregrado aun requieren esfuerzos en varias direcciones. Creemos que es vital el fortalecimiento de cada una de las tres fases del AIE. En la fase virtual pretendemos desarrollar nuevas formas de presentación de la información, que permitan identificar con mayor precisión, los conceptos centrales del contenido temático y su aplicación a la resolución de problemas. Uno de los aspectos que merece atención tiene que ver con la forma de presentación de la información en el entorno virtual (videos). Proyectamos utilizar nuevos formatos de presentación (por ejemplo, lightboard) y comparar los diversos tipos de carga cognitiva que generan para el estudiante con respecto al formato tradicional que estamos utilizando (Power Point, Microsoft).

Igualmente, la crisis mundial actual por el COVID-19, obliga a replantear las sesiones presenciales del aula. Por ejemplo, la imposibilidad de conducir sesiones de discusión en grupos pequeños de trabajo en el aula física nos ha obligado a realizarlas dentro del entorno virtual. Este aspecto representa un punto de inflexión en el concepto tradicional del aula invertida, en el que primero existe un componente virtual para el estudio independiente y luego otro presencial para la interacción.

Por supuesto, los desafíos son mayores cuando se pretende incorporar la tercera fase del AIE (simulación clínica) a la virtualidad. Estos problemas ofrecen oportunidades, no solo para enfrentar la crisis, sino para desarrollar estrategias educativas en el periodo poscrisis. Hacemos un llamado a la comunidad quirúrgica para desarrollar un modelo de AIE en la virtualidad, así como para generar vínculos para el trabajo cooperativo. Otros proyectos para el futuro desarrollo tienen que ver con la integración del entorno clínico al aula invertida y con la aplicación del modelo en la educación de posgrado en cirugía. En particular, pretendemos trasladar el modelo de AIE a la simulación quirúrgica, mediante metodologías de tutoría remota, para el desarrollo de habilidades prácticas entre los médicos residentes.

Finalmente, hemos identificado limitaciones en la investigación educativa. Necesitamos conducir estudios cualitativos que informen a profundidad sobre nuestros resultados. También es necesario involucrar grupos control en la exploración de algunos de los desenlaces, que hasta el momento cuentan únicamente con diseño pre y post-intervención en un solo grupo.

\section{Cumplimiento de normas éticas}

Consentimiento informado. Este artículo se trata de una síntesis de la experiencia local, que no involucra de forma primaria a pacientes como sujetos de investigación, por eso no se diligenció consentimiento informado.

Conflicto de interés. Ninguno declarado por los autores.

Financiación. Este estudio fue financiado con recursos de la Facultad de Medicina de Universidad de La Sabana (Colombia).

Contribución de los autores. Los autores declaran haber contribuido en el desarrollo del estudio. Diseño, 
escritura, revisión y aprobación final del manuscrito: Luis C Domínguez-Torres, Neil V Vega-Peña, Diego Orlando Sierra-Barbosa, Juan José Pepín-Rubio, Juan David Lotero.

\section{Referencias}

1. Lewis CE, Chen DC, Relan A. Implementation of a flipped classroom approach to promote active learning in the third-year surgery clerkship. Am J Surg. 2018;215:298303. https://doi.org/10.1016/j.amjsurg.2017.08.050

2. Liebert CA, Mazer L, Bereknyei Merrell S, Lin DT, Lau JN. Student perceptions of a simulation-based flipped classroom for the surgery clerkship: A mixed-methods study. Surgery. 2016;160:591-8.

https://doi.org/10.1016/j.surg.2016.03.034

3. Liebert CA, Lin DT, Mazer LM, Bereknyei S, Lau JN. Effectiveness of the surgery core clerkship flipped classroom: A prospective cohort trial. Am J Surg. 2016;211:451-457.e1. https://doi.org/10.1016/j.amjsurg.2015.10.004

4. Hew KF, Lo CK. Flipped classroom improves student learning in health professions education: A meta-analysis. BMC Med Educ. 2018;18:38. https://doi.org/10.1186/s12909-018-1144-z.

5. Chen F, Lui AM, Martinelli SM. A systematic review of the effectiveness of flipped classrooms in medical education. Med Educ. 2017;51:585-97. https://doi.org/10.1111/medu.13272

6. Domínguez LC, Vega NV, Espitia EL, Sanabria ÁE, Corso C, Serna AM, Osorio C. Impacto de la estrategia de aula invertida en el ambiente de aprendizaje en cirugía: una comparación con la clase magistral. Biomédica. 2015;35:513-21.

https://doi.org/10.7705/biomedica.v35i4.2640

7. Dominguez LC, Espitia EL, Vega NV, Sanabria ÁE, Osorio $C$, Tarazona N. Outline of an innovative learning environment of surgery: A comparison of students' perceptions with a traditional learning environment based in a DREEM analysis. 3EE2 (20676). In: AMEE 2014 (International Association for medical education) Abstract Book. 2014. p. 197.

8. Domínguez LC, Espitia EL, Vega N V, Sanabria ÁE. Teamwork attitude: not just a student issue. Med Educ. 2015;49:1154-5.

https://doi.org/10.1111/medu.12872

9. Moros G, Domínguez LC, Sierra D, Villarraga A, Pepin JJ. Actividades profesionales confiables en cirugía mínimamente invasiva: iniciando el camino para lograr las competencias en el futuro graduado de medicina. Rev Chil Cir. 2018;70:503-9. https://doi.org/10.4067/s0718-40262018000600503

10. Domínguez LC, Vega V, Sanabria Á, Restrepo J. Construcción de puentes entre la medicina y las humanidades: papel del soporte y la articulación en el aprendizaje en cirugía. Biomédica. 2019;39:55-64.

https://doi.org/10.7705/biomedica.v39i1.4012

11. Vandewaetere M, Manhaeve D, Aertgeerts B, Clarebout G, Van Merriënboer JJG, Roex A. 4C/ID in medical education: How to design an educational program based on whole-task learning: AMEE Guide No. 93. Med Teach. 2015;37:4-20.

https://doi.org/10.3109/0142159X.2014.928407

12. Domínguez LC, Sierra D, Pepín JJ, Moros G, Villarraga A. Efecto del Aula Invertida Extendida a simulación clínica para la resucitación del paciente traumatizado: estudio piloto de las percepciones estudiantiles sobre el aprendizaje. Rev Colomb Anestesiol. 2017;45(Supl 2):4-11. https://doi.org/10.1016/j.rca.2017.07.011

13. Domínguez LC, Vega NV. Efectos del mapa conceptual sobre la síntesis de información en un ambiente de aprendizaje interactivo: Un estudio preexperimental. Educ Médica. 2020;21:193-7.

https://doi.org/10.1016/j.edumed.2018.08.002

14. Domínguez LC, Carreno O, Sierra DO. Divide y vencerás: Efectos de dos intervenciones para el aprendizaje interactivo en grupos grandes de estudiantes sobre la percepción de la calidad del ambiente de aprendizaje. Educ Médica. 2020 (en prensa). https://doi.org/10.1016/j.edumed.2020.04.006

15. Domínguez LC, Sanabria A, Sierra D. ¿Cómo perciben los estudiantes el clima de aprendizaje en el aula invertida en cirugía?: Lecciones aprendidas y recomendaciones para su implementación. Rev Chil Cir. 2018;70:140-6. https://doi.org/10.4067/s0718-40262018000200140

16. Bonnes SL, Ratelle JT, Halvorsen AJ, Carter KJ, Hafdahl LT, Wang AT, et al. Flipping the quality improvement classroom in residency education. Acad Med. 2017;92: 101-7.

https://doi.org/10.1097/ACM.0000000000001412

17. Miles S, Swift L, Leinster SJ. The Dundee Ready Education Environment Measure (DREEM): a review of its adoption and use. Med Teach. 2012;34:e620-34. https://doi.org/10.3109/0142159X.2012.668625

18. Buldu M, Buldu N. Concept mapping as a formative assessment in college classrooms: Measuring usefulness and student satisfaction. Procedia Soc Behav Sci. 2010;2:2099-104. https://doi.org/10.1016/j.sbspro.2010.03.288

19. Stalmeijer RE, Dolmans DHJM, Wolfhagen IHAP, Muijtjens AMM, Scherpbier AJJA. The development of an instrument for evaluating clinical teachers: involving stakeholders to determine content validity. Med Teach. 2008;30:e272-7. https://doi.org/10.1080/01421590802258904

20. Choo SSY, Rotgans JI, Yew EHJ, Schmidt HG. Effect of worksheet scaffolds on student learning in problem-based learning. Adv Health Sci Educ Theory Pract. 2011;16:517-28. https://doi.org/10.1007/s10459-011-9288-1 
21. Fisher M, King J, Tague G. Development of a self-directed learning readiness scale for nursing education. Nurse Educ Today. 2001;21:516-25. https://doi.org/10.1054/nedt.2001.0589

22. Biggs J, Kember D, Leung DYP. The revised two-factor Study Process Questionnaire: R-SPQ-2F. Br J Educ Psychol. 2001;71:133-49. https://doi.org/10.1348/000709901158433

23. Obenrader C, Broome ME, Yap TL, Jamison F. Changing team member perceptions by implementing TeamSTEPPS in an emergency department. J Emerg Nurs. 2019;45:31-7. https://doi.org/10.1016/j.jen.2018.08.006

24. Schraw G, Dennison RS. Assessing metacognitive awareness. Contemp Educ Psychol. 1994;19:460-75. https://doi.org/10.1006/ceps.1994.1033

25. Domínguez LC, Sierra D, Sanabria Á, Restrepo J. ¿Soy parte del equipo?: transformando las percepciones estudiantiles en un instrumento para evaluar el trabajo en equipo en cirugía. Educ Médica. 2019;20:213-20. https://doi.org/10.1016/j.edumed.2018.11.005

26. Domínguez LC, Sanabria ÁE. Validez de constructo y confiabilidad del ROTA-Q para la evaluación de la calidad académica de las rotaciones clínicas en estudiantes de medicina. Educ Méd. 2109;20:71-8. https://doi.org/10.1016/j.edumed.2017.11.010

27. Domínguez LC, Sanabria ÁE, Sierra DO. El clima productivo en cirugía: ¿una condición para el aprendizaje en el aula invertida?. Educ Med. 2018;19(Suppl 3):263-9. https://doi.org/10.1016/j.edumed.2017.08.001

28. Domínguez LC, Alfonso E, Restrepo JA, Pacheco M. Clima de aprendizaje y preparación para el aprendizaje autodirigido en cirugía: ¿influye el enfoque de enseñanza?. Educ Med. 2020;21:84-91. https://doi.org/10.1016/j.edumed.2018.05.006

29. Ruiz-Parra AI, Ángel-Muller E, Guevara O. La simulación clínica y el aprendizaje virtual. Tecnologías complementarias para la educación médica. Rev. Fac. Med. 2009;57:67-79.

30. Bustamante E, Sanabria Á. Evaluación de las actitudes hacia el profesionalismo en estudiantes de medicina. Rev Colomb Cir. 2014;29:222-9.

31. Riveros RE, Espinosa A, Jimenez P, Martinez IV L. E-learning experience: A teaching model with undergraduate surgery students in a developing country. In: Studies in Health Technology and Informatics. 2005. p. 404-6.

32. Angarita FA, Price B, Castelo M, Tawil M, Ayala JC, Torregrossa L. Improving the competency of medical students in clinical breast examination through a standardized simulation and multimedia-based curriculum. Breast Cancer Res Treat. 2019;173:439-45. https://doi.org/10.1007/s10549-018-4993-6

33. Vergel J, Quintero GA, Isaza-Restrepo A, Ortiz-Fonseca M, Latorre-Santos C, Pardo-Oviedo JM. The influence of different curriculum designs on students' dropout rate: a case study. Med Educ Online. 2018;23:1432963. https://doi.org/10.1080/10872981.2018.1432963

34. Isaza-Restrepo A, Pérez-Olmos I, Martínez LE, Velásquez A, Mercado MA. El tiempo de los estudiantes de medicina en las rotaciones clínicas y el sistema de créditos. Rev Cienc Salud. 2006;4:136-46.

35. Quintero GA, Vergel J, Arredondo M, Ariza MC, Gómez P, Pinzon-Barrios AM. Integrated medical curriculum: Advantages and disadvantages. J Med Educ Curric Dev. 2016;3:133-7. https://doi.org/10.4137/JMECD.S18920

36. Bustamante E, Sanabria Á. Spanish adaptation of The Penn State College of Medicine Scale to assess professionalism in medical students. Biomédica. 2014; 34:291-9.

37. Rubiano AM, Sánchez ÁI, Guyette F, Puyana JC. Trauma care training for national police nurses in Colombia. Prehospital Emerg Care. 2010;14:124-30.

https://doi.org/10.3109/10903120903349762

38. Sanabria AE, Jaime-Contreras M, Aristizabal H. Experiencia quirúrgica de la sección cirugía general de la Universidad de Antioquia Hospital San Vicente de Paul. Rev colomb Cir. 2002;17:199-206.

39. Amaya-Arias AC, Barajas R, Eslava-Schmalbach JH, Wheelock A, Gaitán-Duarte H, Hull L, Sevdalis N. Translation, cultural adaptation and content re-validation of the observational teamwork assessment for surgery tool. Int J Surg. 2014;12:1390-402. https://doi.org/10.1016/j.ijsu.2014.10.001

40. Gómez-Ramírez MF, Gómez JC, González-Neira EM, Rúgeles S, Suárez DR, Torregrosa L. Evaluación del mejoramiento de habilidades básicas para cirugía laparoscópica por medio del entrenamiento con un videojuego. Rev Cienc. Salud. 2014;12(Esp.):9-20. https://doi.org/10.12804/revsalud12.esp.2014.02

41. Cervera S, Márquez-Ustáriz A, Sánchez-Cortes E. Evaluación de competencias quirúrgicas en residentes de cirugía general mediante el uso de smartphone. Inv Ed Méd. 2017;6:130. https://doi.org/10.1016/j.riem.2017.01.124

42. Cortés-Murgueitio N, Cuevas L, Díaz-Castrillon CE, Pinzón F, Molina GR. Experiencia y curva de aprendizaje de apendicectomía y colecistectomía laparoscópica de los residentes de cirugía general en un hospital latinoamericano. Universitas Médicas. 2019;60:1-10. https://doi.org/10.11144/Javeriana.umed60-2.curv

43. Herrera-Almario GE, Kirk K, Guerrero VT, Jeong K, Kim $\mathrm{S}$, Hamad GG. The effect of video review of resident laparoscopic surgical skills measured by self- and external assessment. Am J Surg. 2016;211:315-20. https://doi.org/10.1016/j.amjsurg.2015.05.039

44. Vargas LC, Herrera G, Zuchini AM. Aplicación de las redes sociales en la educación del cirujano general. @Cirbosque rompiendo el paradigma. Cir Andal. 2020;31:15-20.

https://doi.org/10.37351/2020311.2 
45. Bermúdez C, Monroy-Melo A, Torregrosa L, Henao-Pérez F. Estado actual de la formación de residentes de cirugía general en Colombia. Rev Colomb Cir. 2006;21:225-39.

46. Rojas D, Cristancho S, Rueda C, Grierson L, Monclou A, Dubrowski A. The validation of an instrumented simulator for the assessment of performance and outcome of knot tying skill: A pilot study. Stud Health Technol Inform. 2011;63:517-23.

47. Sierra-Sierra S, Sierra-Sierra A, Peñaloza-Barrera C, Uribe-Valencia A, Durán-Meléndez MÁ. Diseño de bitácora centrado en el residente: conociendo y mejorando la experiencia de usuario. Rev Colomb Cir. 2018;33:398405. https://doi.org/10.30944/20117582.87

48. Cabrera LF, Luna-Jaspe C, Pedraza-Ciro M. Adaptación de la residencia de Cirugía General en Colombia a la pandemia del COVID-19: programa de enseñanza quirúrgica virtual. Rev Colomb Cir. 2020;35:256-63. https://doi.org/10.30944/20117582.632
49. Sánchez S, Ariza A. Educación quirúrgica en Colombia en la era del COVID-19. Rev Colomb Cir. 2020;35:251-6. https://doi.org/10.30944/20117582.631

50. Henao O, Escallón J, Green J, Farcas M, Sierra JM, Sánchez W, Okrainec A. Fundamentos de cirugía laparoscópica en Colombia con telesimulación: una herramienta adicional para la formación integral de cirujanos. Biomédica. 2013;33:107-14.

https://doi.org/10.7705/biomedica.v33i1.1425

51. Barrera C.A. CA, Salamanca J.D. JD, González Neira E.M. EM, Suárez D.R. D, Rúgeles S. Sistema de evaluación de habilidades psicomotrices en cirujanos de laparoscopia. Rev Cienc Salud. 2016;14(Esp.):57-67.

https://doi.org/10.12804/revsalud14.especial.2016.04

52. Bayter E, Cordoba A, Messier J. Residentes como docentes en la Fundación Universitaria de Ciencias de la Salud (FUCS), proyecto de residentes formadores. Repert Med Cir. 2017;26:27-34.

https://doi.org/10.1016/j.reper.2017.03.001 